\title{
F. MUSY
}

\section{CHARNAY}

\section{Sur le théorème de Stein-Rosenberg}

Revue française d'automatique, informatique, recherche opérationnelle. Analyse numérique, tome 8, no 2 (1974), p. 95-107.

<http://www.numdam.org/item?id=M2AN_1974_8_2_95_0>

(C) AFCET, 1974, tous droits réservés.

L'accès aux archives de la revue "Revue française d'automatique, informatique, recherche opérationnelle. Analyse numérique » implique l'accord avec les conditions générales d'utilisation (http://www.numdam.org/legal. php). Toute utilisation commerciale ou impression systématique est constitutive d'une infraction pénale. Toute copie ou impression de ce fichier doit contenir la présente mention de copyright.

\section{Numdam}

Article numérisé dans le cadre du programme

Numérisation de documents anciens mathématiques

http://www.numdam.org/ 
R.A.I.R.O.

(8e année, août 1974, R-2, p. 95 à 107)

\title{
SUR LE THEOREME DE STEIN-ROSENBERG*
}

\author{
par F. Musy (1) et M. Charnay ( ${ }^{2}$ ) \\ Communiqué par F. ROBERT
}

Résumé. - On présente une extension du théorème de Stein-Rosenberg qui l'éclaire d'un nouveau point de vue : A étant une matrice carrée à éléments $\geqslant 0$, on peut comparer son rayon spectral avec celui d'un produit $A J_{r} \ldots A_{j_{1}}$ de matrices « extraites 》 de $A$ et de la matrice unité.

\section{INTRODUCTION}

Rappelons la formulation classique du :

\section{Théorème de Stein-Rosenberg [2] [4]}

Soit $A=L+U$ une matrice carrée à éléments $\geqslant 0$, de diagonale nulle, de triangulaire inférieure stricte $L$, et de triangulaire supérieure $U$.

Seuls les trois cas suivants (mutuellement exclusifs) sont possibles :

a)

$$
0 \leqslant \rho\left[(I-L)^{-1} U\right] \leqslant \rho(L+U)<1
$$

b)

$$
\rho(L+U)=\rho\left[(I-L)^{-1} U\right]=1
$$

c)

$$
1<\rho(L+U) \leqslant p\left[(I-L)^{-1} U\right]
$$

$\rho$ désigne la fonction rayon spectral : maximum des modules des valeurs propres.

On rappelle que ce théorème règle la comparaison des méthodes de Jacobi et Gauss Seidel dans le "cas nonnégatif » pour la résolution itérative de systèmes linéaires [4].

L'étude de procédés itératifs chaotiques pour la résolution de systèmes non linéaires [3] nous a amenés à élaborer un résultat qui constitue en fait une extension du théorème ci-dessus en l'éclairant d'un nouveau point de vue.

Le but de cet article est de mettre en forme cette extension.

(1) Ecole Centrale de Lyon.

(2) S.A.N.T.I.-U.E.R. de Mathématiques Lyon I.

Revue Française d'Automatique, Informatique et Recherche Opérationnelle nº août 1974, R-2. 
Une matrice $A$ à éléments $\geqslant 0$ sera dite nonnégative $(A \geqslant 0)$. Un vecteur $u$ de $R^{n}$ à composantes $\geqslant 0$ sera noté $u \geqslant 0$ et $u>0$ si toutes ses composantes sont $>0$; un vecteur $u$ nonnégatif et différent de 0 sera noté $: \geqslant 0$.

Soit $A$ une matrice $(n, n)$ nonnégative, et $J$ une partie non vide de $\{1,2, \ldots, n\}$. On définit la matrice (nonnégative) $A_{J}$ de la façon suivante :

pour $j \in J A_{J}$ a même $j^{\text {ème }}$ ligne que $A$

pour $j \notin J A_{J}$ a même $j^{\text {ème }}$ ligne que la matrice unité $(n, n)$.

EXEMPLE : $n=4 \quad J=\{2,4\} \quad A=\left(a_{i j}\right)$. Alors

$$
A_{J}=\left(\begin{array}{llll}
1 & 0 & 0 & 0 \\
a_{21} & a_{22} & a_{23} & a_{24} \\
0 & 0 & 1 & 0 \\
a_{41} & a_{42} & a_{43} & a_{44}
\end{array}\right)
$$

\section{RESULTATS PRELIMINAIRES}

La proposition suivante montre comment, dans le théorème de SteinRosenberg, la matrice $(I-L)^{-1} U$ peut être obtenue à partir de $A=L+U$, comme produit de matrices du type défini ci-dessus.

\section{Proposition 1.}

Soit $A=L+U$ une matrice $(n, n)$ nonnégative de diagonale nulle, de triangulaire inférieure stricte $L$ (et de triangulaire supérieure $U$ ). Alors $(I-L)^{-1} U$ s'écrit :

$$
(I-L)^{-1} U=A_{\{n\}} \ldots A_{\{2\}} \cdot A_{\{1\}}
$$

La démonstration de cette identité, purement algébrique, ne présente aucune difficulté.

\section{EXEMPLE :}

$$
\begin{aligned}
& n=3 \\
& A=\left[\begin{array}{lll}
0 & 2 & 3 \\
5 & 0 & 4 \\
6 & 7 & 0
\end{array}\right]=\left[\begin{array}{lll}
0 & & U \\
& 0 \\
L & 0
\end{array}\right] \\
& (I-L)^{-1} U=\left[\begin{array}{rrr}
0 & 2 & 3 \\
0 & 10 & 19 \\
0 & 82 & 151
\end{array}\right]=\left[\begin{array}{lll}
1 & 0 & 0 \\
0 & 1 & 0 \\
6 & 7 & 0
\end{array}\right]\left[\begin{array}{lll}
1 & 0 & 0 \\
5 & 0 & 4 \\
0 & 0 & 1
\end{array}\right]\left[\begin{array}{lll}
0 & 2 & 3 \\
0 & 1 & 0 \\
0 & 0 & 1
\end{array}\right]
\end{aligned}
$$


Notre but est de montrer que le théorème de Stein-Rosenberg s'applique en fait à d'autres produits de matrices $A_{J}$ que celui qui vient d'être mis en évidence.

Nous utiliserons pour cela le corollaire «fin » suivant du théorème classique de Perron-Frobenius.

Proposition 2 : [1] [2] [3] [5].

Soit $A=\left(a_{i j}\right)$ une matrice nonnégative $(n, n)$. Pour $u>0$ et $v: \geqslant 0$ dans $R^{n}$, on pose :

$$
\eta_{A}(v)=\operatorname{Min}_{\substack{i=1,2 \ldots \ldots \\ v i \neq 0}} \sum_{j=1}^{n} a_{i j} \frac{v_{j}}{v_{i}} \quad ; \quad \tau_{A}(u)=\operatorname{Max}_{i=1,2 \ldots n} \sum_{j=1}^{n} a_{i j} \frac{u_{j}}{u_{i}}
$$

Alors

$$
\operatorname{Sup}_{v \neq \geqslant 0} \eta_{A}(v)=\rho(A)=\operatorname{Inf}_{u>0} \tau_{A}(u) .
$$

(L'intérêt de ce corollaire est d'être vrai que $A$ soit ou non irréductible.)

\section{LE RESULTAT PRINCIPAL}

Nous l'énonçons dans le :

Théorème 1 : Soit $A$ une matrice nonnégative $(n, n)$. Soit $J_{1}, J_{2} \ldots J_{r}$ une séquence de parties non vides de $\{1,2, \ldots n\}$ dont l'union redonne $\{1,2, \ldots n\}$ :

$$
\bigcup_{i=1}^{r} J_{i}=\{1,2, \ldots, n\}
$$

(On ne suppose pas nécessairement les $J_{i}$ disjoints deux à deux). On pose

$$
K=A_{J_{r}} \cdot A_{J_{r-1}} \cdot \ldots \cdot A_{J_{1}}
$$

Alors seuls les trois cas suivants (mutuellement exclusifs) sont possibles.

a)

$$
\begin{array}{ll}
\text { a) } & 0 \leqslant \rho(A)^{r} \leqslant \rho(K) \leqslant \rho(A)<1 \\
\text { b) } & \rho(A)=\rho(K)=1 \\
\text { c) } & 1<\rho(A) \leqslant \rho(K) \leqslant \rho(A)^{r}
\end{array}
$$

c)

$1^{\circ}$ Etablissons tout d'abord le point $a$ ).

Pour cela, démontrons un premier résultat intermédiaire. 


\section{Lemme 1 :}

Soit $A$ une matrice nonnégative $(n, n)$ de rayon spectral $\rho(A)<1$. Soit $u>0$ dans $R^{n}$ tel que $\tau_{A}(u)<1$ (un tel $u$ existe d'après la proposition 2). Soit $T=A_{J_{p}} \ldots A_{J_{2}} \cdot A_{J_{1}}$ où $J_{1}, J_{2}, \ldots J_{p}$ est une séquence de parties non vides de $\{1,2, \ldots n\}$. Soient $t_{i j}$ les éléments de $T$.

Alors

$$
\sum_{j=1}^{n} t_{i j} \frac{u_{j}}{u_{i}} \leqslant \tau_{A}(u) \quad \text { pour } \quad i \in J_{1} \cup J_{2} \ldots \cup J_{p}
$$

Preuve du lemme 1.

Elle se fait par récurrence.

Pour $p=1$ et pour $i$ pris dans $J_{1}$, il vient par définition :

$$
\sum_{j=1}^{n}\left[A_{J 1}\right]_{i j} \frac{u_{j}}{u_{i}}=\sum_{j=1}^{n} a_{i j} \frac{u_{j}}{u_{i}} \leqslant \tau_{A}(u)
$$

Posons $S=A_{J_{p-1}} \cdot A_{J_{p-2}} \quad \ldots A_{J 1}$.

Supposons, pour hypothèse de récurrence, $s_{i j}$ étant les éléments de $S$, que :

$$
\sum_{j=1}^{n} s_{i j} \frac{u_{j}}{u_{i}} \leqslant \tau_{A}(u) \quad \text { pour } \quad i \in J_{1} \cup J_{2} \ldots \cup J_{p-1}
$$

et montrons que

$$
\sum_{j=1}^{n} t_{i j} \frac{u_{j}}{u_{i}} \leqslant \tau_{A}(u) \quad \text { pour } \quad i \in J_{1} \cup J_{2} \ldots \cup J_{p}
$$

Soit d'abord $i \in J_{p}$. Puisque $T=A_{J_{p}} \cdot S$, on a :

$$
\sum_{j=1}^{n} t_{i j} \frac{u_{j}}{u_{i}}=\sum_{j=1}^{n}\left(\sum_{l=1}^{n} a_{i l} s_{l j}\right) \frac{u_{j}}{u_{i}}=\sum_{l=1}^{n}\left(\sum_{j=1}^{n} s_{l j} \frac{u_{j}}{u_{i}}\right) a_{i l}
$$

Or le produit à gauche par une matrice $A_{J}$ ne perturbe que les lignes d'indices pris dans $J$. A l'aide de cette remarque, on démontre aisément que pour tout indice $j$ pris dans $\{1,2, \ldots, n\}$ et n'appartenant pas à $J_{1} \cup J_{2} \ldots \cup J_{p-1}$, la $j^{\text {ème }}$ ligne de $S$ est la $j$ ème ligne de la matrice unité.

Donc :

$$
\sum_{j=1}^{n} t_{i j} \frac{u_{j}}{u_{i}}=\sum_{l \xi J_{1}} \sum_{J_{2} . . \cup_{J_{p-1}}} a_{i l} \frac{u_{l}}{u_{i}}+\sum_{l \in J_{1}} \sum_{J_{2} \ldots}\left(\sum_{j=1}^{n} s_{l j} \frac{u_{j}}{u_{i}}\right) a_{i l}
$$

Grâce à l'hypothèse de récurrence, on déduit l'inégalité :

$$
\sum_{j=1}^{n} t_{i j} \frac{u_{j}}{u_{i}} \leqslant \sum_{l i J_{1} \cup_{J_{2} \ldots} \ldots \cup_{J_{p-1}}} a_{i l} \frac{u_{l}}{u_{i}}+\tau_{A}(u) \sum_{l \in J_{1}} \sum_{J_{2} \ldots \cup_{J_{p-1}}} a_{i l} \frac{u_{l}}{u_{i}}
$$

Revue Française d'Automatique, Informatique et Recherche Opérationnelle 
D'où a fortiori, puisque $\tau_{A}(u)<1$

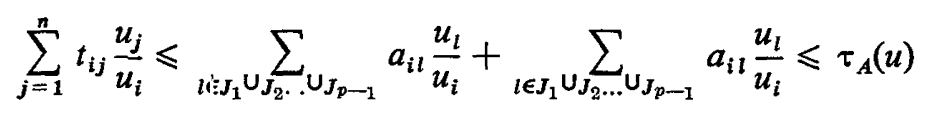

Supposons maintenant que

$$
i \in J_{1} \cup J_{2} \ldots \cup J_{p-1} \quad \text { et } \quad i \notin J_{p}
$$

Puisque $i \notin J_{p}$ la $i^{\text {ème }}$ ligne de $A_{J_{p}}$ est $\left[\begin{array}{llllll}0 & \ldots & 0 & 1 & 0 & \ldots\end{array}\right]$, par définition de $A_{J_{p}}$ et puisque $T=A_{J_{p}} \cdot S$, il est clair que $T$ et $S$ ont même $i^{\text {ème }}$ ligne. Donc :

$$
\sum_{j=1}^{n} t_{i j} \frac{u_{j}}{u_{i}}=\sum_{j=1}^{n} s_{i j} \frac{u_{j}}{u_{i}} \leqslant \tau_{A}(u)
$$

ce qui achève la démonstration du lemme 1 .

En appliquant ce résultat à la matrice $K=A_{J_{r}} \ldots A_{J_{2}} \cdot A_{J_{1}}$ et puisque $J_{1} \cup J_{2} \ldots \cup J_{r}=\{1,2, \ldots, n\}$, on obtient, $k_{i j}$ étant les éléments de $K$ :

$$
\sum_{j=1}^{n} k_{i j} \frac{u_{j}}{u_{i}} \leqslant \tau_{A}(u)
$$

pour tout $u>0$ tel que $\tau_{A}(u)<1$ et pour tout $i=1,2, \ldots, n$.

D'où

$$
\tau_{K}(u)=\operatorname{Max}_{i=1,2 \ldots, n} \sum_{j=1}^{n} k_{i j} \frac{u_{j}}{u_{i}} \leqslant \tau_{A}(u)
$$

D'où encore

$$
\operatorname{Inf}_{u>0} \tau_{K}(u) \leqslant \operatorname{Inf}_{\substack{u>0 \\ \tau_{A}(u)<1}} \tau_{K}(u) \leqslant \operatorname{Inf}_{\substack{u>0 \\ \tau_{A}(u)<1}} \tau_{A}(u)=\operatorname{Inf}_{u>0} \tau_{A}(u)
$$

c'est-à-dire, d'après la proposition 2 :

$$
\rho(K) \leqslant \rho(A)
$$

Une partie du point $a$ ) est démontrée. Le reste de la démonstration est une conséquence du

$\mathrm{n}^{\circ}$ août 1974, R-2. 


\section{Lemme 2 :}

Soit $A$ une matrice nonnégative $(n, n)$ de rayon spectral $\rho(A) \leqslant 1$. Soit $\omega \geqslant 0$ un secteur propre de $A$ attaché à la valeur propre $\rho(A)$. (Théorème de Perron-Frobenius [5]).

Soit $T=A_{J_{p}} \ldots A_{J_{2}} \cdot A_{J_{1}}$ où $J_{1}, J_{2} \ldots J_{p}$ est une séquence de parties non vides de $\{1,2, \ldots, n\}$.

Soit $J=\left\{j \in\{1,2, \ldots, n\} / \omega_{j} \neq 0\right\}$.

Alors

$$
\sum_{j=1}^{n} t_{i j} \frac{\omega_{j}}{\omega_{i}} \geqslant \rho(A)^{p} \quad \text { pour } \quad i \in J \cap\left(J_{1} \cup J_{2} \ldots \cup J_{p}\right)
$$

\section{Preuve du lemme 2.}

Elle se fait par une récurrence analogue à la précédente. $p=1 \bullet$ Soit $i \in J \cap J_{1}$ supposé non vide (sinon il n'y a rien à démontrer).

$$
\sum_{j=1}^{n}\left[A_{J 1}\right]_{i j} \frac{\omega_{j}}{\omega_{i}}=\sum_{j=1}^{n} a_{i j} \frac{\omega_{j}}{\omega_{i}}=\rho(A)
$$

On pose à nouveau $S=A_{J_{p-1}} \ldots A_{J_{2}} \cdot A_{J_{1}}$

On suppose que

$$
\sum_{j=1}^{n} s_{i j} \frac{\omega_{j}}{\omega_{i}} \geqslant \rho(A)^{p-1} \quad \text { pour } \quad i \in J \cap\left(J_{1} \cup J_{2} \ldots \cup J_{p-1}\right),
$$

et on démontre que :

$$
\sum_{j=1}^{n} t_{i j} \frac{\omega_{j}}{\omega_{i}} \geqslant \rho(A)^{p} \quad \text { pour } \quad i \in J \cap\left(J_{1} \cup J_{2} \ldots \cup J_{p}\right)
$$

Soit $i \in J \cap J_{p}$ supposé non vide.

Il vient en utilisant le calcul précédent :

$$
\sum_{j=1}^{n} t_{i j} \frac{\omega_{j}}{\omega_{i}}=\sum_{l \in J_{1}} \sum_{U_{2} \ldots \cup_{J_{p-1}}} a_{i l} \frac{\omega_{l}}{\omega_{i}}+\sum_{l \in J_{1}} \sum_{J_{2} \ldots U_{J p-1}}\left(\sum_{j=1}^{n} s_{l,} \frac{\omega_{j}}{\omega_{i}}\right) a_{i l}
$$

D'où :

$$
\sum_{j=1}^{n} t_{i j} \frac{\omega_{j}}{\omega_{i}} \geqslant \sum_{l\left(J_{1} \cup \cup_{J_{2}} \ldots \cup_{J_{p-1}}\right.} a_{i l} \frac{\omega_{l}}{\omega_{i}}+\sum_{l \in J \cap\left(J_{1} U_{J_{2}} \ldots \cup_{\left.J_{p-1}\right)}\right.}\left(\sum_{j=1}^{n} s_{l j} \frac{\omega_{j}}{\omega_{i}}\right) \cdot a_{i l}
$$

Grâce à l'hypothèse de récurrence, on a :

$$
\sum_{j=1}^{n} t_{i j} \frac{\omega_{j}}{\omega_{i}} \geqslant \sum_{l i: J_{1} \cup \sum_{J_{2} \ldots} \ldots U_{J_{p-1}}} a_{i l} \frac{\omega_{l}}{\omega_{i}}+\sum_{l \in J \cap\left(J_{1} \cup J_{J_{2} \ldots} \cup_{J_{p}-1}\right)} a_{i l} \frac{\omega_{l}}{\omega_{i}} \rho(A)^{p-1}
$$


d'où

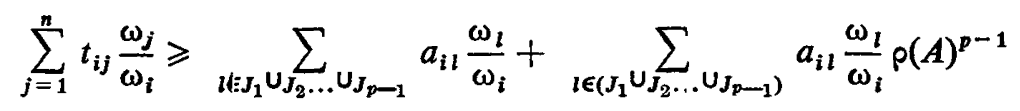

puisque

$$
\sum_{l \in J n\left(J_{1} \cup J_{2} \ldots \cup_{J_{p-1}}\right)} a_{i l} \frac{\omega_{l}}{\omega_{i}}=\sum_{l \in\left(J_{1} \cup U_{J_{2} \ldots} \ldots U_{J_{p-1}}\right)} a_{i l} \frac{\omega_{l}}{\omega_{i}}
$$

Donc

$$
\begin{aligned}
\sum_{j=1}^{n} t_{i j} \frac{\omega_{j}}{\omega_{i}} \geqslant \sum_{l=1}^{n} a_{i l} \frac{\omega_{l}}{\omega_{i}}-\left(\sum_{l \in J_{1} U_{. J} J_{p-1}} a_{i l} \frac{\omega_{l}}{\omega_{i}}\right)\left(1-\rho(A)^{p-1}\right) \\
\geqslant \rho(A)-\rho(A)\left(1-\rho(A)^{p-1}\right)=\rho(A)^{p}
\end{aligned}
$$

D'autre part si $J \cap\left(J_{1} \cup J_{2} \ldots \cup J_{p-1}\right) \neq \varnothing$ et pour $i \in J \cap\left(J_{1} \cup J_{2} \ldots \cup J_{p-1}\right)$ et $i \notin J_{p}$, on a encore :

$$
\sum_{j=1}^{n} t_{i j} \frac{\omega_{j}}{\omega_{i}}=\sum_{j=1}^{n} s_{i j} \frac{\omega_{j}}{\omega_{i}} \geqslant \rho(A)^{p-1} \geqslant \rho(A)^{p}
$$

et le lemme 2 est établi. On l'applique à la matrice $K$, d'où :

$$
\sum_{j=1}^{n} k_{i j} \frac{\omega_{j}}{\omega_{i}} \geqslant \rho(A)^{r} \quad \text { pour } \quad i \in J
$$

Donc $\rho(A)^{r} \leqslant \eta_{K}(\omega) \leqslant \rho(K)$ et $\left.a\right)$ est entièrement démontré.

$2^{\circ}$ On établit de façon analogue les points $b$ ) et $c$ ) par l'intermédiaire des deux lemmes suivants.

\section{Lemme 3 :}

Soit $A$ une matrice nonnégative $(n, n)$ de rayon spectral $\rho(A) \geqslant 1$. Soient $\varepsilon$ réel $>0$ donné et $u>0$ dans $R^{n}$ tel que : $\tau_{A}(u)<\rho(A)+\varepsilon$ [un tel $u$ existe d'après la proposition 2].

Soit $T=A_{J_{p}} \ldots A_{J_{2}} \cdot A_{J_{1}}$ ou $J_{1}, J_{2} \ldots J_{p}$ est une séquence de parties non vides de $\{1,2, \ldots, n\}$. Alors :

$$
\sum_{j=1}^{n} t_{i j} \frac{u_{j}}{u_{i}} \leqslant(\rho(A)+\varepsilon)^{p}
$$

pour $i \in J_{1} \cup J_{2} \ldots \cup J_{p}$.

$n^{\circ}$ août 1974, R-2. 
Preuve du lemme 3.

Là aussi on mène une récurrence tout à fait semblable aux précédentes. Notons $p=\rho(A)$ $p=1$. Le résultat est évident.

On pose

On suppose que

$$
S=A_{J_{p-1}} \ldots A_{J_{2}} \cdot A_{J_{1}}
$$

$$
\sum_{j=1}^{n} s_{i j} \frac{u_{j}}{u_{i}} \leqslant(\rho+\varepsilon)^{p-1}
$$

pour $i \in J_{1} \cup J_{2} \ldots \cup J_{p-1}$.

Soit $i \in J_{p}$.

$$
\sum_{j=1}^{n} t_{i j} \frac{u_{j}}{u_{i}}=\sum_{l \ell J_{1} \cup_{J_{2}} \ldots U_{J_{p-1}}} a_{i l} \frac{u_{l}}{u_{i}}+\sum_{l \in J_{1} \cup \bigcup_{J_{2} \ldots} \cup_{J_{p-1}}}\left(\sum_{j=1}^{n} s_{l j} \frac{u_{j}}{u_{i}}\right) a_{i l}
$$

D'où :

$$
\sum_{j=1}^{n} t_{i j} \frac{u_{j}}{u_{i}} \leqslant \sum_{l\left(J_{1} \cup \bigcup_{J_{2} \ldots} \ldots \cup_{J_{p-1}}\right.} a_{i l} \frac{u_{l}}{u_{i}}+(\rho+\varepsilon)^{p-1}\left(\sum_{l \in J_{1} \cup J_{2} \ldots U_{J_{p-1}}} a_{i l} \frac{u_{l}}{u_{i}}\right)
$$

On décompose

$$
\sum_{i=1}^{n} a_{i i} \frac{u_{l}}{u_{i}} \text { en } \sum_{l i J_{1} \cup J_{J_{2} \ldots} \cup_{J_{p-1}}} a_{i i} \frac{u_{l}}{u_{i}}+\sum_{t \in J_{1} \cup} \sum_{J_{2} \ldots} a_{U_{J_{p-1}}} \frac{u_{l}}{u_{i}}
$$

D'où encore :

$$
\begin{aligned}
& \sum_{j=1}^{n} t_{i j} \frac{u_{j}}{u_{i}} \leqslant \sum_{l=1}^{n} a_{i l} \frac{u_{l}}{u_{i}}-\sum_{l \in J_{1}} \sum_{J_{2} \ldots U_{J_{p-1}}} a_{i l} \frac{u_{l}}{u_{i}} \\
& +(\rho+\varepsilon)^{p-1}\left(\sum_{l \in J_{1} \cup J_{2} \ldots \cup_{J_{p-1}}} a_{i l} \frac{u_{l}}{u_{i}}\right) \\
& \sum_{j=1}^{n} t_{i j} \frac{u_{j}}{u_{i}} \leqslant \sum_{i=1}^{n} a_{i l} \frac{u_{l}}{u_{i}}+\left(\sum_{l \in J_{1} \cup J_{2} \ldots \cup_{J_{p-1}}} a_{i l} \frac{u_{l}}{u_{i}}\right)\left[(\rho+\varepsilon)^{p-1}-1\right] \\
& \sum_{j=1}^{n} t_{i j} \frac{u_{j}}{u_{i}} \leqslant \sum_{i=1}^{n} a_{i l} \frac{u_{l}}{u_{i}}+\left(\sum_{l=1}^{n} a_{i l} \frac{u_{l}}{u_{i}}\right)\left[(\rho+\varepsilon)^{p-1}-1\right] \\
& \sum_{j=1}^{n} t_{i j} \frac{u_{j}}{u_{i}} \leqslant\left(\sum_{l=1}^{n} a_{i l} \frac{u_{l}}{u_{i}}\right)\left(1+(\rho+\varepsilon)^{p-1}-1\right) \\
& =\left(\sum_{l=1}^{n} a_{i l} \frac{u_{l}}{u_{i}}\right)(\rho+\varepsilon)^{p-1} \leqslant(\rho+\varepsilon)(\rho+\varepsilon)^{p-1}=(\rho+\varepsilon)^{P}
\end{aligned}
$$


D'autre part si $i \in J_{1} \cup J_{2} \ldots \cup J_{p-1}$ et $i \notin J_{p}$

$$
\sum_{j=1}^{n} t_{i j} \frac{u_{j}}{u_{i}}=\sum_{j=1}^{n} s_{i j} \frac{u_{j}}{u_{i}} \leqslant(\rho+\varepsilon)^{p-1}<(\rho+\varepsilon)^{p}
$$

ce qui achève la démonstration du lemme 3 .

\section{Lemme 4 :}

Soit $A$ une matrice non négative $(n, n)$ de rayon spectral $\rho(A) \geqslant 1$. Soit $\omega \geqslant 0$ un vecteur propre de $A$ attaché à la valeur propre $\rho(A)$.

Soit $T=A_{J_{p}} \cdot \ldots \cdot A_{J_{2}} \cdot A_{J_{1}}$ ou $J_{1}, J_{2}, \ldots, J_{p}$ est une séquence de parties non vides de $\{1,2, \ldots, n\}$.

Soit $J=\left\{j \in\{1,2, \ldots, n\} / \omega_{j} \neq 0\right\}$.

Alors

$$
\sum t_{i j} \frac{\omega_{j}}{\omega_{i}} \geqslant \rho(A) \quad \text { pour } \quad i \in J \cap\left(J_{1} \cup J_{2} \cup \ldots \cup J_{p}\right)
$$

La démonstration de ce lemme s'effectue en utilisant une technique analogue à celle du lemme 2 . C'est pourquoi elle n'est pas détaillée.

En appliquant les lemmes 3 et 4 à la matrice $K$, on a :

si $\rho(A) \geqslant 1, \sum_{j=1}^{n} k_{i j} \frac{\omega_{j}}{\omega_{i}} \geqslant \rho(A)$ pour $i \in J$ grâce au lemme 4.

D'où

$$
\rho(K)=\operatorname{Sup}_{v \geqslant 0} \eta_{K}(v) \geqslant \eta_{K}(\omega)=\operatorname{Min}_{i \in J} \sum_{j=1}^{n} k_{i j} \frac{\omega_{j}}{\omega_{i}} \geqslant \rho(A) \geqslant 1
$$

et grâce au lemme 3 :

$$
\operatorname{Max}_{i=1,2, \ldots, n} \sum_{j=1}^{n} k_{i j} \frac{u_{j}}{u_{i}} \leqslant(\rho+\varepsilon)^{r}
$$

pour tout $u>0$ tel que $\tau_{A}(u)<\rho+\varepsilon$ (en notant $\rho(A)=\rho$ ).

D'où

$$
\rho(K)=\operatorname{Inf}_{u>0} \tau_{K}(u) \leqslant \operatorname{Inf}_{\substack{u>0 \\ \tau_{\Delta}(u)<p+\varepsilon}} \tau_{K}(u) \leqslant(\rho+\varepsilon)^{r}
$$

Ainsi $\rho(K) \leqslant(\rho+\varepsilon)^{r}$ et ceci pour tout $\varepsilon>0$; c'est-à-dire $\rho(K) \leqslant \rho^{r}$. En résumé : $\rho \leqslant \rho(K) \leqslant \rho^{r}$ et dans le cas particulier où $\rho=1$, on obtient $\rho(K)=1$.

La démonstration du théorème 1 est complète. $n^{\circ}$ août 1974, R-2. 


$$
\begin{aligned}
& \text { EXEMPLE : } n=4 \quad J_{1}=\{1,2\} \quad J_{2}=\{1,3\} \quad J_{3}=\{4\} \\
& A=\left(\begin{array}{llll}
0,2 & 0,1 & 0,3 & 0,1 \\
0,1 & 0 & 0,1 & 0,1 \\
0,2 & 0,3 & 0,1 & 0,2 \\
0,2 & 0,1 & 0,4 & 0
\end{array}\right) \\
& K=A_{J_{3}} \cdot A_{J_{2}} \cdot A_{J_{1}}=\left(\begin{array}{llll}
1 & 0 & 0 & 0 \\
0 & 1 & 0 & 0 \\
0 & 0 & 1 & 0 \\
0,2 & 0,1 & 0,4 & 0
\end{array}\right)\left(\begin{array}{llll}
0,2 & 0,1 & 0,3 & 0,1 \\
0 & 1 & 0 & 0 \\
0,2 & 0,3 & 0,1 & 0,2 \\
0 & 0 & 0 & 1
\end{array}\right) \text {. } \\
& \cdot\left[\begin{array}{llll}
0,2 & 0,1 & 0,3 & 0,1 \\
0,1 & 0 & 0,1 & 0,1 \\
0 & 0 & 1 & 0 \\
0 & 0 & 0 & 1
\end{array}\right]=\left(\begin{array}{llll}
0,05 & 0,02 & 0,37 & 0,13 \\
0,10 & 0 & 0,10 & 0,10 \\
0,07 & 0,02 & 0,19 & 0,25 \\
0,045 & 0,012 & 0,16 & 0,136
\end{array}\right)
\end{aligned}
$$

On vérifie que l'on a

$$
\rho(A)^{3}=0,215<\rho(K)=0,457 \ldots<\rho(A)=0,599 \ldots<1 .
$$

La proposition suivante montre donc dans quel cas le produit $A_{J_{r}} \ldots A_{J_{2}} \cdot A_{J_{1}}$ peut s'écrire sous la forme $(I-L)^{-1} U$ avec $A=L+U$.

\section{Proposition 3 :}

Soit $A$ une matrice $(n, n)$ nonnégative.

Soit $J_{1}, J_{2}, \ldots, J_{r}$ une séquence de parties non vides de $\{1,2, \ldots, n\}$ formant une partition de $\{1,2, \ldots, n\}$ :

$$
J_{1} \cup J_{2} \ldots \cup J_{r}=\{1,2, \ldots, n\} ; \quad J_{i} \cap J_{j}=\varnothing \quad \text { pour } i \neq j .
$$

Soit $K=A_{J_{r}} \ldots A_{J_{2}} \cdot A_{J_{1}}$. On peut construire, alors, deux matrices

$$
L \geqslant 0 \text { et } U \geqslant 0
$$

telles que :

$$
\begin{gathered}
A=L+U \\
(I-L) K=U
\end{gathered}
$$

et comme $\rho(L)=0, K=(I-L)^{-1} U \geqslant 0$. 
On va construire $L$ et $U$ par leurs éléments respectifs $l_{i j}$ et $u_{i j}$.

Soit $i \in\{1,2, \ldots, n\}$; il existe $j$ unique dépendant de $i$ tel que $i \in J_{j}$ puisque les $J_{s}, s=1,2, \ldots, n$ forment une partition de $\{1,2, \ldots, n\}$.

Posons alors :

pour $s \in J_{j} \cup J_{j+1} \ldots \cup J_{r}$

$$
\begin{aligned}
u_{i s} & =a_{i s} \\
l_{i s} & =0
\end{aligned}
$$

pour $s \notin J_{j} \cup J_{j+1} \ldots \cup J_{r}$

$$
\begin{aligned}
u_{i s} & =0 \\
l_{i s} & =a_{i s}
\end{aligned}
$$

On vérifie que $L$ et $U$ sont nonnégatives et que $A=L+U$. D'autre part, on remarque que pour tout $i \in J_{j}$ :

$$
k_{i s}=\left[A_{J_{r}} \ldots A_{J_{2}} \cdot A_{J_{1}}\right]_{i s}=\left[A_{J_{r-1}} \ldots A_{J_{2}} \cdot A_{J_{1}}\right]_{i s}=\ldots=\left[A_{J_{1}} \ldots A_{J_{2}} \cdot A_{J_{1}}\right]_{i s}
$$

Donc toujours pour $i \in J_{j}$ :

$k_{i s}=\sum_{l \in J_{j}, \cup_{J_{i+1} \ldots} \cup_{J_{r}}} a_{i l} \cdot\left[A_{J_{i-1}} \ldots A_{J_{2}} A_{J_{1}}\right]_{l s}+a_{i s}$ pour $s \in J_{j} \cup J_{j+1} \ldots \cup J_{r}$ et

$$
k_{i s}=\sum_{l \Downarrow J_{j} \cup_{J_{j}+1} \ldots \cup_{J_{r}}} a_{i l} \cdot\left[A_{J_{i-1}} \ldots A_{J_{2}} A_{J_{1}}\right]_{l s} \quad \text { pour } \quad s \notin J_{j} \cup J_{j+1} \ldots \cup J_{r}
$$

Donc pour $i \in J_{j}$

$$
k_{i s}=\sum_{l i J_{j} \cup \cup_{j+1} \ldots \cup \cup_{J_{r}}} a_{i l} k_{l s}+a_{i s} \quad \text { pour } \quad s \in J_{j} \cup J_{j+1} \ldots \cup J_{r}
$$

et

$$
k_{i s}=\sum_{l \notin J j} \sum_{J_{j}+1} \ldots \cup_{J_{r}} a_{i l} k_{l s} \quad \text { pour } \quad s \notin J_{j} \cup J_{j+1} \ldots \cup J_{r}
$$

ce qui s'écrit encore d'après la définition de $L$ et $U$ :

$$
K=L K+U \quad \text { soit }(I-L) K=U
$$

Il est facile de voir que la matrice $L$ construite par ce procédé peut s'écrire sous la forme $L=P S P^{t}$ où $S$ est une matrice nonnégative triangulaire inférieure stricte et $P$ une matrice de permutation. Donc $\rho(L)=0$ et $L$ étant non négative, $I-L$ est une $M$ matrice et $K=(I-L)^{-1} U \geqslant 0$.

no août 1974, R-2. 


$$
\begin{aligned}
& \text { EXEMPLE : } n=4 \quad J_{1}=\{2,4\} \quad J_{2}=\{3\} \quad J_{3}=\{1\} \\
& A=\left[\begin{array}{rrrr}
1 & 2 & 3 & 4 \\
5 & 6 & 7 & 8 \\
9 & 10 & 11 & 12 \\
13 & 14 & 15 & 16
\end{array}\right] L=\left[\begin{array}{rrrr}
0 & 2 & 3 & 4 \\
0 & 0 & 0 & 0 \\
0 & 10 & 0 & 12 \\
0 & 0 & 0 & 0
\end{array}\right] U=\left[\begin{array}{rrrr}
1 & 0 & 0 & 0 \\
5 & 6 & 7 & 8 \\
9 & 0 & 11 & 0 \\
13 & 14 & 15 & 16
\end{array}\right]
\end{aligned}
$$$$
A=L+U \quad \text { et } \quad \rho(L)=0<1
$$

$$
\begin{aligned}
K=A_{J_{3}} \cdot A_{J_{2}} \cdot A_{J_{1}}= & {\left[\begin{array}{rrrr}
1 & 2 & 3 & 4 \\
0 & 1 & 0 & 0 \\
0 & 0 & 1 & 0 \\
0 & 0 & 0 & 1
\end{array}\right]\left[\begin{array}{rrrr}
1 & 0 & 0 & 0 \\
0 & 1 & 0 & 0 \\
9 & 10 & 11 & 12 \\
0 & 0 & 0 & 1
\end{array}\right] . } \\
& \cdot\left[\begin{array}{rrrr}
1 & 0 & 0 & 0 \\
5 & 6 & 7 & 8 \\
0 & 0 & 1 & 0 \\
13 & 14 & 15 & 16
\end{array}\right]=\left[\begin{array}{rrrr}
708 & 752 & 857 & 896 \\
5 & 6 & 7 & 8 \\
215 & 228 & 261 & 272 \\
13 & 14 & 15 & 16
\end{array}\right]
\end{aligned}
$$

On vérifie que l'on a :

$$
\begin{gathered}
K=(I-L)^{-1} U \text { avec d'ailleurs (théorème 1) } \\
1<\rho(A)=36,2<\rho(K)=990,2<\rho(A)^{3}=47,5 \cdot 10^{3} .
\end{gathered}
$$

REMARQUe : la proposition 3 reste vraie si $A$ est une matrice réelle $(n, n)$ quelconque. Toutefois les matrices $L, U$ et $(I-L)^{-1} U$ ne sont plus nonnégatives.

\section{CAS PARTICULIER}

Soit $A$ une matrice nonnégative $(n, n)$. Considérons la partition de $\{1,2, \ldots, n\}$ en $n$ parties $J_{j}=\{j\}(j=1,2, \ldots, n)$. Si l'on construit les matrices $L$ et $U$ de la proposition 3, $L$ est alors la triangulaire inférieure stricte de $A$ et $U$ la triangulaire supérieure de $A$. cer le

La proposition 3 redonne la proposition 1 et le théorème 1 permet d'énon- 
Théorème 2 : Soit $A$ une matrice nonnégative $(n, n)$ de triangulaire inférieure stricte $L$ et de triangulaire supérieure $U$. Seuls les trois cas (mutuellement exclusifs) sont possibles.

a) $0 \leqslant \rho(L+U)^{n} \leqslant \rho\left((I-L)^{-1} U\right) \leqslant \rho(L+U)=\rho(A)<1$

b) $\rho(A)=\rho(L+U)=\rho\left((I-L)^{-1} U\right)=1$

c) $1<\rho(A)=\rho(L+U) \leqslant \rho\left((I-L)^{-1} U\right) \leqslant \rho(L+U)^{n}$

Ce résultat est à rapprocher de la version provenant du théorème de "Stein-Rosenberg tronqué » [6]. Notons toutefois que les inégalités introduisant $\rho(A)^{n}$ sont nouvelles. Notons aussi que dans la proposition 1 et le théorème de Stein-Rosenberg classique, la nullité de la diagonale de $A$ est une condition parfaitement anecdotique : elle peut être purement et simplement supprimée.

\section{REFERENCES}

[1] C. Odiard, Un corollaire du théorème de Perron-Frobenius. R.I.R.O., 1971, R. 2, pages 124 à 129 .

[2] F. RoberT, Cours de D.E.A., Université de Lyon 1 (1973).

[3] F. ROBERT, M. ChARNAY et F. Musy, Itérations chaotiques série-parallèle pour des équations non linéaires de point fixe (à paraître dans Num. Math.).

[4] R. S. VARGa, Matrix Iterative Analysis, Prentice Hall (1962).

[5] R. S. VARGa, On a Connexion between Informa of Normes and eigenvalues of associated operators. Linear Algebra and its Applications, 6, 249-256 (1973).

[6] F. ROBERT, Algorithmes tronqués de découpe linéaire, R.I.R.O., 1972, R. 2, pages 45 à 64 . 